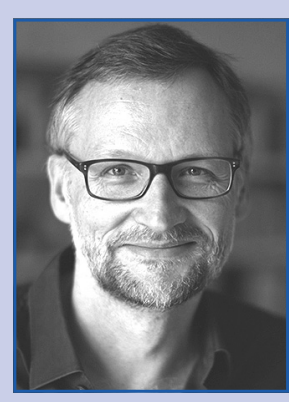

Prof. Dr. Ralph Hertwig ist Direktor des Forschungsbereichs Adaptive Rationalität am Max-Planck-Institut für Bildungsforschung.

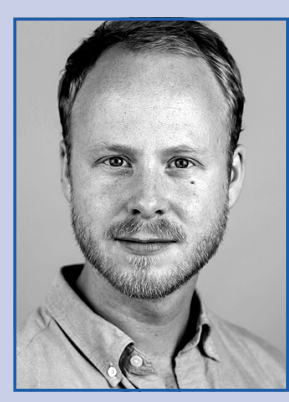

Dipl.-Psych. Dirk U. Wulff ist Doktorand am Forschungsbereich Adaptive Rationalität am Max-Planck-Institut für Bildungsforschung.

\title{
Risikoentscheidungen: Die Kluft zwischen Erfahrung und Beschreibung
}

\author{
Ralph Hertwig und Dirk U. Wulff
}

Dieser Artikel gibt eine Einführung in das "Description-Experience Gap" der Beobachtung eines systematischen Unterschieds in Risikoentscheidungen aufgrund symbolischer Beschreibungen bzw. sequentieller Erfahrungen. Die "Kluft" wird besonders deutlich bei seltenen Ereignissen, die zu viel Gewicht (Beschreibung) bzw. zu wenig Gewicht (Erfahrung) erhalten. Ursachen und Implikationen werden diskutiert.

\section{Das "Gewicht" seltener Ereignisse}

In den Jahren nach 2007 rang die Welt mit der Gefahr einer Kernschmelze des internationalen Finanzsystems. Mehrere extreme Ereignisse, von denen jedes für sich genommen sehr unwahrscheinlich schien - der Zusammenbruch der U.S. Bank Lehman Brothers, der drohende Bankrott großer Banken (z. B.: Hypo Real Estate), großer Finanzdienstleister (z. B.: AIG, Fannie Mae, Freddie Mac) und ganzer Staaten -, folgten in schneller Abfolge aufeinander. Was als eine Krise des internationalen Finanzsystems begann, wurde schnell zu einer Weltwirtschaftskrise, in deren Folge globale Unternehmen in Schieflage gerieten, Staatsbudgets aus dem Ruder liefen und die Arbeitslosigkeit in vielen Ländern rasant anstieg. Warum war die Welt auf die Möglichkeit dieser Ereignisse so schlecht vorbereitet? Eine Erklärung lautet, dass die Risikomanagementmodelle mit ihrer auf der Normalverteilungshypothese aufbauenden Logik in jenen Welten scheitern, die systematisch von der Gauß-Funktion abweichen (z. B. „fat tails"-Verteilungen). In Welten, in denen selten doch nicht so selten ist wie theoretisch angenommen, unterschätzen diese Modelle die Häufigkeit seltener, extremer Verluste (vgl. Taleb, 2007).

Das angeblich so optimierte Risikomanagement ist aber sicher nicht das alleinige Glied in der Ursachenkette. Auch die individuellen Marktteilnehmer haben sich offensichtlich nicht genügend auf das Risiko extremer ökonomischer Ereignisse eingestellt - zum Beispiel all jene
Besitzer einer Immobilie, die im Verlauf der Krise ihre Kredite nicht mehr bedienen konnten und sich mit einer Zwangsversteigerung konfrontiert sahen. Warum aber haben viele Akteure durchweg die Möglichkeit seltener, extremer Ereignisse unterschätzt oder gar völlig ignoriert? Können psychologische Theorien und Befunde - letztere gewonnen in Verhaltensexperimenten $\mathrm{zu}$ Entscheidungen unter Risiko und Unsicherheit - diese mutmaßliche Achtlosigkeit gegenüber seltenen, aber schwerwiegenden Ereignissen erklären? Auf den ersten Blick nicht. Zahlreiche Studien der Entscheidungsforschung scheinen eher eine gegenteilige Tendenz zu belegen: Die Wahrscheinlichkeit seltener Ereignisse wird häufig überschätzt. Zum Beispiel werden relativ seltene Risiken, wie die einer Lebensmittelvergiftung oder Lungenkrebs infolge von Nikotingenuss, im Schnitt viel zu hoch eingeschätzt (vgl. Lichtenstein et al., 1978; Viscusi, 2002). Mediale Berichterstattung, die dazu neigt, seltenen, aber dramatischen Krankheiten und Todesursachen (z. B. BSE und die Creutzfeld-JakobKrankheit) unverhältnismäßig viel Beachtung zu schenken, spielt bei dieser Tendenz zur Überschätzung sicher eine Rolle (vgl. Renn, 2014). Aber selbst wenn seltene Risiken nicht geschätzt werden müssen, sondern explizit quantifiziert sind, wird unwahrscheinlichen Ereignissen mehr psychologisches Gewicht beigemessen als ihnen, gemessen an ihrer objektiven Wahrscheinlichkeit, zustünde. Dies ist zumindest eine der zentralen Annahmen in der Cumulative ProspectTheorie (vgl. Tversky/Kahneman, 1992), der einflussreichsten deskriptiven Theorie des Entscheidens unter Risiko. Mithil- 
fe der Annahme einer nichtlinearen Wahrscheinlichkeitsgewichtungsfunktion, die einen umgekehrt $S$-förmigen Verlauf nimmt und nach der seltene Ereignisse „übergewichtet“ und Ereignisse mit mittleren und hohen Wahrscheinlichkeiten „untergewichtet“ werden, erklärt sie Verhaltensanomalien, die die Erwartungsnutzentheorie vor Probleme stellt (z. B.: gleichzeitige Risikofreude und Risikoaversion in Gestalt von Lottospielen und Erwerb von Versicherungen).

Warum also handeln Menschen, die nach weithin akzeptierter Sichtweise dazu neigen, geringe Wahrscheinlichkeiten zu überschätzen oder diesen, sobald sie expliziert werden, zu viel Gewicht einzuräumen, als ob sie die Möglichkeit seltener katastrophaler Ereignisse nicht ernst genug nähmen? Um dieses scheinbare Paradox zu verstehen, hilft eine Unterscheidung, die seit etwa einer Dekade in der Entscheidungsforschung zunehmend Beachtung findet: Über die Wahrscheinlichkeit eines unsicheren Ereignisses kann man - sehr vereinfacht - auf zwei grundsätzlich unterschiedlichen Wegen Kenntnis erlangen: durch die symbolische Beschreibung seiner Wahrscheinlichkeit oder durch die sequentielle Erfahrung des Auftretens bzw. Nichtauftretens des Ereignisses (vgl. Hertwig et al., 2004). Die Annahme der Überbewertung seltener Ereignisse lässt außer Acht, dass eine Vielzahl von Entscheidungen nicht auf expliziten und beschriebenen Informationen über Wahrscheinlichkeiten beruhen. In diesen Fällen, die nicht die Ausnahme, sondern eher die Regel darstellen, können Menschen häufig nichts anderes tun, als sich auf ihre Erfahrungen zu verlassen.

Ziel dieses Beitrages ist es, Erkenntnisse der psychologischen Forschung zur „Kluft" zwischen erfahrungs- und beschreibungsbasierten Risikoentscheidungen zu erläutern, und Konsequenzen für das unternehmerische Risikomanagement zu diskutieren. Im nächsten $\mathrm{Ab}$ schnitt beschreiben wir hierzu die Grundlagen erfahrungs- und beschreibungsbasierter Risikoentscheidungen. In Abschnitt 3 stellen wir die experimentelle Methodik vor, die verwendet wird, um diese systematische Kluft zwischen den beiden Formaten zu untersuchen. Anschließend umreißen wir in Abschnitt 4 zwei wesentliche Erklärungen für den Unterschied zwischen den beiden Klassen von Entscheidungen. Zum Abschluss veranschaulichen wir in Abschnitt 5 anhand zweier Beispiele im Bereich Konsumenten- und Investitionsentscheidungen mögliche Implikationen für das Controlling.

\section{Erfahrungsbasierte und beschreibungsbasierte Entscheidungen}

Entscheidungsforscher, gleichgültig ob in der Psychologie oder der Ökonomie, untersuchen die Frage, wie Menschen mit Risiko umgehen, häufig mit dem Werkzeug monetärer Lotterien. Die Lotterie, so die Annahme, ist das perfekte Double für reale Entscheidungssituationen. Genau wie Lotterien sind die realen Optionen, zwischen denen es zu entscheiden gilt, nichts anderes als Wahrscheinlichkeitsverteilungen über $n$ mögliche monetäre (und/oder nicht-monetäre) Ausgänge. Daher unterstellen Entscheidungsforscher, wenn sie Personen dabei zusehen, wie sie sich zwischen Lotterien entscheiden, wie diese sich entscheiden würden, wenn es um die Wahl zwischen verschiedenen Investitionsfonds, Partnern oder Karrierewegen ginge. Man kann diese für die Entscheidungsforschung so zweckdienliche Annahme aus vielen Gründen kritisieren. Ein wichtiger Kritikpunkt ist allerdings dieser: Selbst wenn man die Rollenzuschreibung, wonach die Auswahl zwischen Lotterien das Sinnbild für Wahlentscheidungen schlechthin ist, akzeptieren würde, ist ein Aspekt besonders fragwürdig: Lotterien im Labor bestehen im Regelfall aus zwei oder mehreren Optionen und jede Option ist vollständig expliziert, das heißt, die möglichen monetären Ereignisse und dazugehörigen Wahrscheinlichkeiten werden vollständig beschrieben. Abb. 1 illustriert eine einfache und vollständig beschriebene Lotterie mit zwei Optionen: Eine der Optionen ist ein sicheres Ereignis ( $3 €$ mit Sicherheit), die andere Option offeriert eine relativ beträchtliche Summe von $32 €$ mit einer geringen Wahrscheinlichkeit von $10 \%$ oder das Ereignis $0 €$ mit einer Wahrscheinlichkeit von $90 \%$.

Den Luxus, die Konsequenzen und Wahrscheinlichkeiten der Entscheidungsoptionen auf einem Silbertablett dargeboten zu bekommen, gibt es aber nur selten außerhalb des Labors. Das bedeutet jedoch nicht, dass reale Entscheidungen notwendigerweise ohne eine Vorstellung von den zugrunde liegenden möglichen Ereignissen und Wahrscheinlichkeiten getroffen würden. Nicht selten stehen Erfahrungen - die eigenen oder die anderer Personen - in ähnlichen Situationen zur Verfügung. Und manchmal kann man auch die Entscheidungsoptionen erst explorieren, bevor die Entscheidung getroffen wird. Wein, den man beim Erzeuger kauft, kann man zum Beispiel vorher probieren. Oder man kann die Erfahrung anderer mit einem Hotel, einem Sportstudio oder einer Kindergrippe zu Rate ziehen, bevor man sich für ein Angebot entscheidet. Entscheidungen, die auf Basis begrenzter und abzählbarer Erfahrungen mit einer Option getroffen werden, nennen wir erfahrungsbasierte Entscheidungen (Decisions from experience). Im Gegensatz dazu nennen wir Entscheidungen, für die eine vollständige Beschreibung der möglichen Ereignisse und ihrer Wahrscheinlichkeiten vorliegt, beschreibungsbasierte Entscheidungen (Decisions from description). Beschreibungen können symbolische oder graphische Form annehmen.

Die Analyse beschreibungs- und erfahrungsbasierter Entscheidungen existierte lange unabhängig voneinander. Erst in den letzten zehn Jahren hat sich dies grundlegend geändert. Die Initialzündung lieferte die Entdeckung eines systematischen und gravierenden Unterschieds in den Entscheidungen, die auf der Grundlage von Beschreibung und Erfahrung getroffen werden: Die „Kluft“ zwischen beschreibungs- und erfahrungsbasierten Entscheidungen (Description-experience gap; vgl. Hertwig/Erev, 2009).

\section{Die Kluft zwischen beschrei- bungs- und erfahrungsbasierten Entscheidungen}

Wie kann man das in Abb. 1 dargestellte Entscheidungsparadigma in ein erfah-

$\begin{aligned} & \text { Wähle zwischen } \\ & \text { A: } 3\end{aligned}$ mit Sicherheit
oder
$\begin{array}{lll}\text { B: } 32 & \text { mit Wahrscheinlichkeit 10\% } \\ & 0 & \text { mit Wahrscheinlichkeit } 90 \%\end{array}$
Abb. 1: Eine typische beschreibungsbasierte
Lotterie mit zwei Optionen




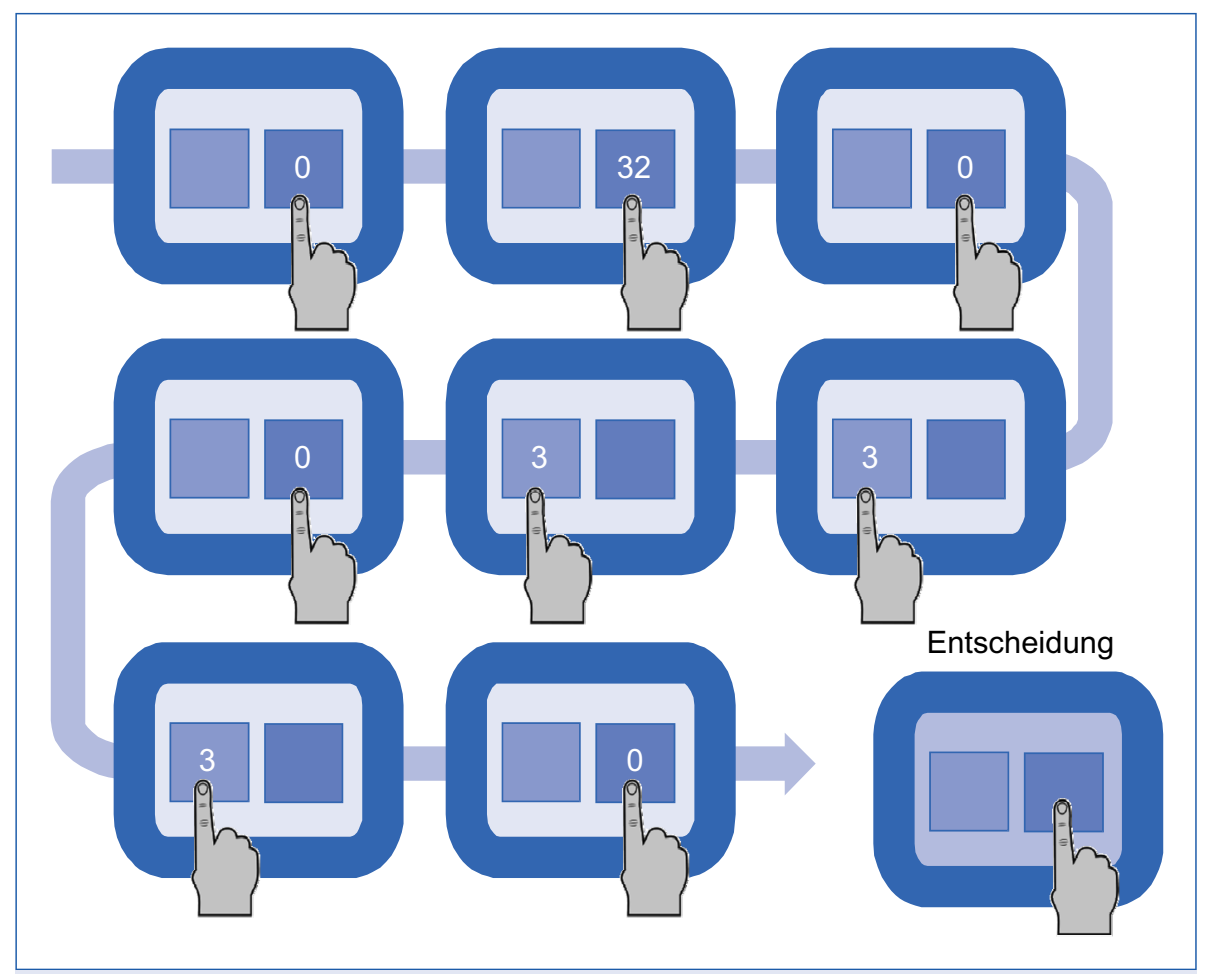

Abb. 2: Schematische Darstellung des Sampling-Paradigmas zur Untersuchung von entscheidungsbasierten Risikowahlen

rungsbasiertes Paradigma übersetzen, und zwar so, dass beide Situationen, zumindest im Prinzip, die gleichen Informationen zur Verfügung stellen? Entscheidungsforscher bedienen sich dazu der folgenden Anordnung: Versuchspersonen sehen zwei (oder mehr) Kästchen auf einem Computerbildschirm. Diese repräsentieren zwei zu Beginn völlig unbekannte Wahrscheinlichkeitsverteilungen. Das Klicken auf die Kästchen löst jeweils eine Zufallsziehung eines möglichen Ereignisses gemäß der zugrunde liegenden Verteilung aus. Basierend auf dieser Anordnung kann man drei Paradigmen unterscheiden. Im Sampling-Paradigma (Abb. 2; vgl. Hertwig et al., 2004) können Versuchspersonen so viele $\mathrm{Zu}$ fallsziehungen auslösen, wie sie möchten, bevor sie die Exploration der Verteilungen beenden. Dann werden sie gebeten, sich zu entscheiden, auf welche Option sie setzen wollen. Diese letzte Wahl wird am Ende des Experiments ausgespielt und das Ergebnis bestimmt den monetären Gewinn oder Verlust der Person.

Das Sampling-Paradigma stellt eine Umwelt dar, in der die Suche nach Information möglich ist, ohne dass die zufällig gezogenen Ereignisse bereits zu materiellen Gewinnen oder Verlusten führen. Das heißt, die Informationssuche zieht keine Kosten nach sich (mit Ausnahme von Opportunitätskosten). Daher könnte man die Verteilungen sorgfältig explorieren, bevor man eine Entscheidung trifft so wie man sich beispielsweise sehr genau die Speisekarten von zwei teuren Gourmettempeln anschaut, bevor man sich für einen entscheidet. Im Sampling-Paradig$m a$ ist jener Zielkonflikt suspendiert, der unvermeidlich in Umwelten auftritt, in denen man sich zwischen unbekannten Optionen entscheiden muss: Soll man auf das Lernen neuer Informationen und langfristige Gewinnmaximierung setzen oder auf die Maximierung des Gewinns, basierend auf der Grundlage bekannter Informationen (exploration-exploitation tradeoff; vgl. Gupta et al., 2006). Das Sampling-Paradigma lässt sich jedoch schnell in ein Paradigma verändern, in dem dieser Zielkonflikt unvermeidbar ist. Das Partial-Feedback-Paradigma folgt der gleichen Logik wie das Sampling-Paradigma, mit dem einzigen Unterschied, dass nun jede Zufallsziehung (und deren Gesamtanzahl wird jetzt fixiert) bereits zum monetären Endergebnis beiträgt (d. h. dieses reduziert oder erhöht). Das dritte und letzte Paradigma ist das Feedback-Paradigma. Es ist so strukturiert wie das Partial-Feedback-Paradigma, nur dass jetzt nach jeder Zufallsziehung Informationen darüber gegeben werden, welcher Gewinn oder Verlust eingetreten wäre, wenn die andere Verteilung gewählt worden wäre.

Bei der vergleichenden Analyse von beschreibungs- und erfahrungsbasierten Entscheidungen stehen diese drei Paradigmen und die folgende Frage im Fokus: Findet man unter Beschreibung (vgl. Abb. 1) und Erfahrung ein ähnliches oder systematisch unterschiedliches Entscheidungsverhalten? Abb. 3 illustriert beispielhaft die Antwort, die in einer Vielzahl von Studien gefunden wurde. Erfahrungs- und beschreibungsbasierte Formate führen nicht $\mathrm{zu}$ identischem Verhalten. Der Unterschied wird besonders deutlich, wenn eine Entscheidung zwischen einer riskanten Option, die entweder einen relativ hohen Gewinn oder hohen Verlust mit geringer Wahrscheinlichkeit $(<20 \%$; das seltene Ereignis) bietet, und einer sicheren Option, welche mit Sicherheit nur einen moderaten Gewinn oder Verlust garantiert, ansteht. Vor diese Wahl gestellt, fällt in allen drei erfahrungsbasierten Paradigmen die Wahl der Mehrheit auf die riskante Option, sofern das seltene Ereignis unattraktiv ist, aber auf die sichere Option, sofern das seltene Ereignis attraktiv ist (vgl. Abb. 3). Bei beschreibungsbasierten Entscheidungen findet sich die nahezu umgekehrte Mehrheitspräferenz. Insgesamt lässt sich der Unterschied so zusammenfassen: In erfahrungsbasierten Situationen entscheiden sich Menschen so, als ob der Einfluss der seltenen Ereignisse untergewichtet würde (relativ zu den objektiven Wahrscheinlichkeiten), während bei beschreibungsbasierten Entscheidungen ihr Einfluss übergewichtet zu sein scheint.

\section{Was verursacht die Kluft zwischen beschreibungs- und erfahrungsbasierten Entschei- dungen?}

Es gibt mehrere Faktoren, die als verantwortlich für den Unterschied zwischen Erfahrung und Beschreibung sind. Die wichtigsten sind die frugale Informationssuche und die Art und Weise, wie die verfügbare Information verarbeitet wird. Beide Faktoren werden im Folgenden näher erläutert. 


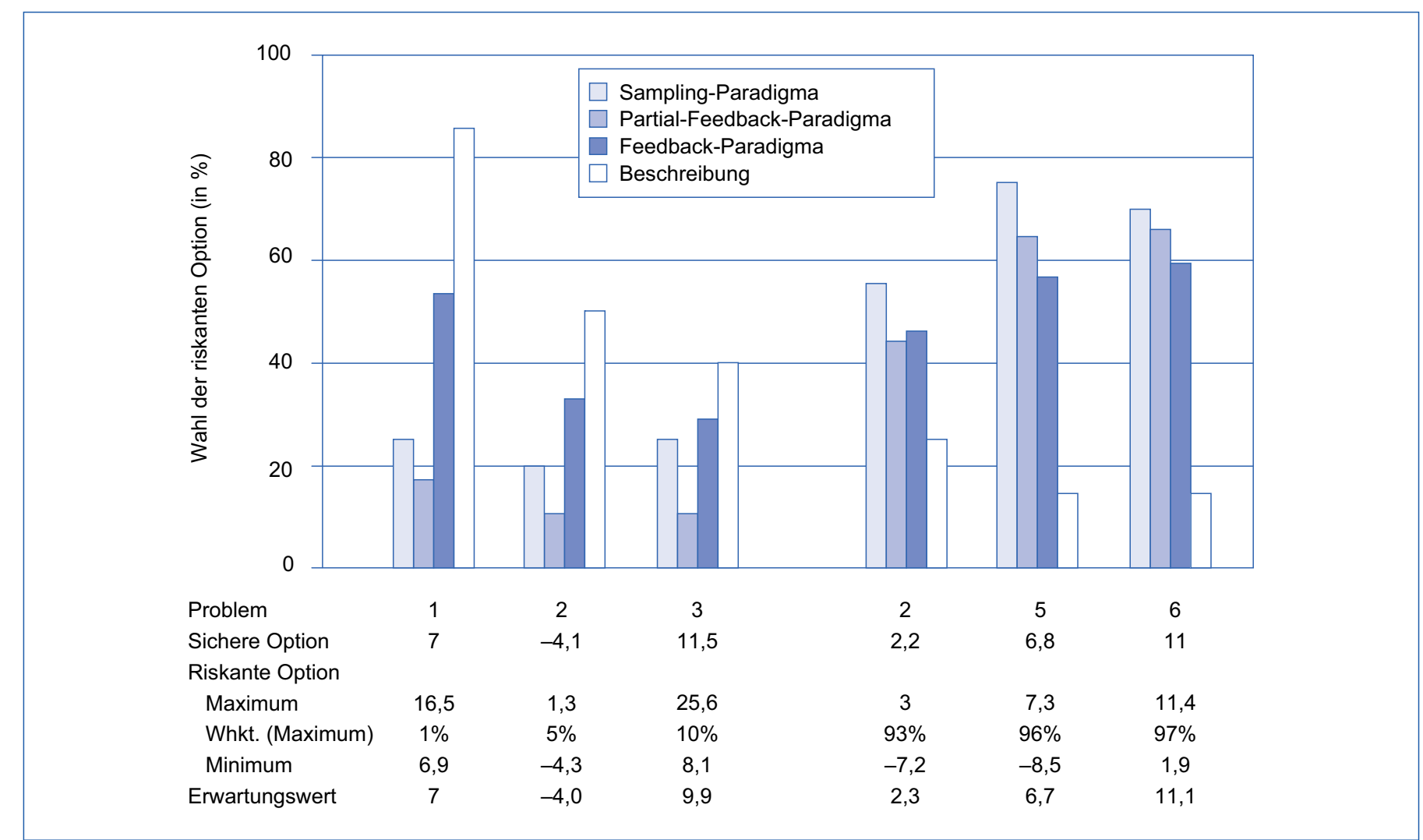

Abb. 3: Prozentsatz von Wahlen der mit Risiko behafteten Option in sechs verschiedenen Lotterien und als Funktion der vier experimentellen Paradigmen (entnommen aus Hertwig/Erev, 2009)

\section{Frugale Suche}

Das Sampling-Paradigma (vgl. Abb. 2) stellt es jeder Person frei, die Optionen sorgfältig zu explorieren (d. h. Zufallsstichproben zu ziehen). Im Durchschnitt findet man allerdings, dass die Anzahl der Ziehungen erstaunlich begrenzt ist. Abb. 4 zeigt die Ergebnisse einer Meta-

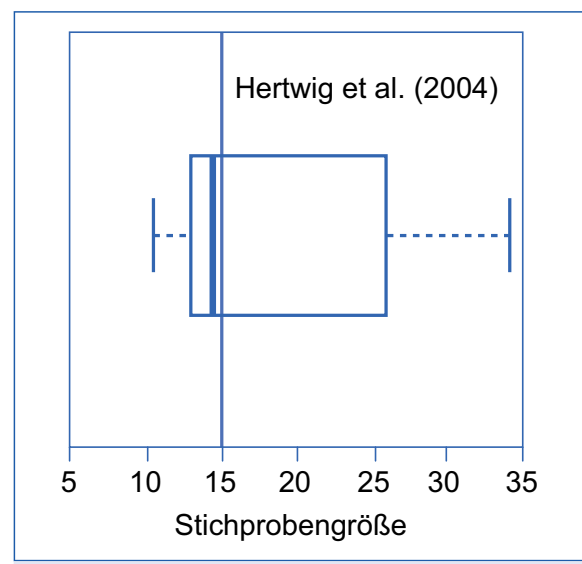

Abb. 4: Verteilung der Stichprobengrößen im Sampling-Paradigma (Ergebnisse aus 21 unabhängigen Datensätzen, basierend auf einer laufenden Metaanalyse von Dirk Wulff und Ralph Hertwig) analyse von über 10.000 Entscheidungen, die von über 1.000 Versuchspersonen stammen. Der Median der Stichprobengröße in der Metaanalyse liegt sehr nahe an dem Median, der bereits in der ersten Untersuchung mit dem Sample-Paradigma beobachtet wurde (Median $=15$; vgl. Hertwig et al., 2004). Im Durchschnitt zeigen sich Versuchspersonen mit relativ wenigen Ziehungen zufrieden und treffen bereits nach etwa $7 \pm 2$ Beobachtungen pro Option eine Entscheidung.

Welche Konsequenzen hat diese relativ frugale Exploration? Stichprobenbasierte Schätzungen über die Wahrscheinlichkeit, mit der Ereignisse auftreten, sind mit einem Schätzfehler behaftet. Dieser Schätzfehler wird, ceteris paribus, desto grösser, je kleiner die Stichprobe ist. Im Extremfall könnte eine kleine Stichprobengröße sogar bedeuten, dass ein seltenes Ereignis darin überhaupt nicht zutage tritt und in der Entscheidung unberücksichtigt bleiben muss. Unterstellt man zum Bespiel ein seltenes Ereignis mit einer Wahrscheinlichkeit von $10 \%$, dann beträgt die „Gefahr“, dass das Ereignis in einer Stichprobe von sieben Ziehungen überhaupt nicht erscheint, beina- he $50 \%$. Aber selbst wenn das Ereignis auftritt, kann eine kleine Stichprobe die Häufigkeit dieses Ereignisses trotzdem „unterrepräsentieren.“ Das hängt mit einer Besonderheit des zugrunde liegenden stochastischen Prozesses zusammen. Die Verteilung der Häufigkeiten eines Ereignisses, beschrieben durch die Binomialverteilung, ist für kleine Wahrscheinlichkeiten (das Ereignis ist selten) und kleine Stichproben schief. Dies bedeutet, dass im Aggregat kleine Erfahrungsstichproben zu Entscheidungen führen können, in denen es mehr Leute gibt, die dem seltenen Ereignis zu wenig Gewicht beimessen, als Leute, die ihm zu viel Gewicht beimessen (im Hinblick auf seine objektive Wahrscheinlichkeit) - entweder weil es überhaupt nicht beobachtet wurde oder weil es durch das Bullauge einer kleinen Stichprobe noch seltener erscheint, als es objektiv ist.

Warum verlassen sich Menschen auf kleine Stichproben? Bevor dieses Verhalten zu schnell als ,irrational“ abgehakt wird, sei Folgendes angemerkt. Die Analyse einer Computersimulation mit 1.000 (zufällig generierten) Lotterieproblemen zeigte, dass ein Agent mit lediglich sieben 
Ziehungen aus jeder Option (14 insgesamt) im Schnitt bereits eine $81 \%$ ige Chance hat, die Option mit dem höheren Erwartungswert zu erkennen. Danach nimmt der Zugewinn an Information durch weitere Ziehungen schnell ab (vgl. Hertwig/Pleskac, 2010). Die Nutzung frugaler Stichproben könnte also auch in der richtigen Intuition begründet sein, dass wenige Ziehungen bereits einen großen Informationsgehalt haben und der marginale Zugewinn weiterer Ziehungen schnell immer kleiner wird. Menschen können eine solche intuitive Kosten-Nutzen-Abwägung treffen. Darauf deutet die Beobachtung hin, dass eine deutliche Steigerung des monetären Anreizes, die attraktivere Option zu erkennen, auch zu deutlich mehr Exploration führt (vgl. Hau et al., 2008). Dies ist aber bei Weitem nicht der einzige Faktor, der die Länge der Informationssuche und damit die Kluft zwischen erfahrungs- und beschreibungsbasierten Entscheidungen beeinflusst. Weitere Faktoren sind beispielsweise die Anwesenheit „wachsamer" Emotionen (wie z. B. Furcht), das Aspirationsniveau einer Person sowie das Alter (und damit die abnehmenden kognitiven Kapazitäten; vgl. Hertwig, im Druck).

\section{Informationsverarbeitung}

Neben der frugalen Suche gibt es einen zweiten Faktor, der gleichfalls zu einer augenscheinlichen "Untergewichtung“ seltener Ereignisse in erfahrungsbasierten Entscheidungen beiträgt. In beschreibungsbasierten Entscheidungen stehen alle Informationen gleichzeitig zur Verfügung (vgl. Abb. 1). Erfahrungsbasierte Informationen reihen sich entlang einer Zeitschiene auf (vgl. Abb. 2). Dieser Formatunterschied ermöglicht eine andere Integration der Information, womöglich bedingt er sie sogar (vgl. Hertwig, im

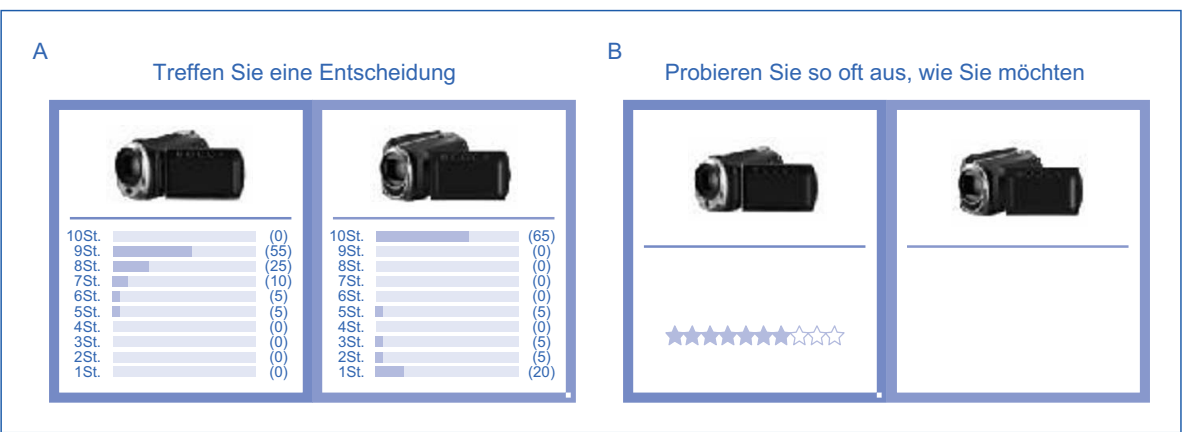

Abb. 5: Beschreibungsbasierte (A) vs. erfahrungsbasierte (B) Konsumentenbewertungen (entnommen aus Wulff et al., 2014)
Druck). Zum Zeitpunkt der Entscheidung müssen beispielsweise die einzelnen Ziehungen oder eine komprimierte Form davon aus dem Gedächtnis abgerufen werden. Häufig materialisiert sich hier der sogenannte Recency-Effekt: Jüngst (recent) gesammelte Informationen erhalten ein höheres Gewicht in der Entscheidung als länger zurückliegende Informationen. Dies könnte zum einen mit durch die Funktionsweise unseres Gedächtnisses verursacht sein: Länger zurückliegende Ereignisse werden in der Regel schlechter erinnert als jüngere Ereignisse. Zum anderen könnte der Recency-Effekt eine adaptive Reaktion auf Umwelten sein, die häufig nichtstationär und kompetitiv sind. Unabhängig von seiner Verursachung führt der Recency-Effekt zu einer „Unterrepräsentation“ seltener Ereignisse (siehe Binomialverteilung), da die ohnehin schon kleine Stichprobe (siehe oben) sich durch diesen Gedächtnisfilter Recency noch weiter verengt.

Die Liste der psychologischen Faktoren, die die Kluft zwischen beschreibungsund erfahrungsbasierten Entscheidungen forcieren, ließe sich noch fortsetzen und auch auf das Partial-Feedback-Paradigma und Feedback-Paradigma ausdehnen (vgl. de Palma et al., im Druck). Die Liste umfasst emotionale und motivationale Voraussetzungen des Entscheidenden, nicht zuletzt aber auch den Einfluss von Suchstrategien. Nicht nur die Menge der gesammelten Information, sondern auch die Suchstrategie (d. h. die Art und Weise, wie nach ihr gesucht wird) scheint unsere Entscheidungen $\mathrm{zu}$ determinieren (vgl. Hills/Hertwig, 2010).

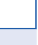

(1)

\section{Welche praktischen Implika- tionen hat die Kluft zwischen beschreibungs- und erfahrungs- basierten Entscheidungen?}

Viele Entscheidungen erlauben nur erfahrungsbasierte Entscheidungen, weil die Handlungsalternativen sich nicht in Gestalt tabellierter Konsequenzen und assoziierter Wahrscheinlichkeiten präsentieren. In Zeiten von Big Data und Einkaufen im Internet stehen uns allerdings immer häufiger Beschreibungen in Form von aggregierten Erfahrungen anderer zur Verfügung. Routine ist dies beispielsweise bereits im Bereich des electronic commerce. Online-Plattformen wie zum Beispiel Amazon oder Ebay offerieren Bewertungstools. Nach dem Erwerb eines Produkts können Konsumenten quantitative Bewertungen beispielsweise in Form von Sternen abgeben. Diese Bewertungen wiederum helfen anderen potenziellen Kunden, die Qualität des Produkts (des Anbieters) einzuschätzen. Häufig spiegelt sich in den Bewertungen ein heterogenes Meinungsbild, was es nahelegt, diese Urteile in ein Gesamtbild zu integrieren. Dafür stehen dem Interessierten oft sowohl die individuellen Urteile als auch eine aggregierte Gesamtschau zur Verfügung. Auf Amazon.com zum Beispiel werden alle Bewertungen in Form eines Balkendiagramms komprimiert (ähnlich wie in Abb. 5, A). Dieses kann dann die Grundlage einer beschreibungsbasierten Produktentscheidung sein. Alternativ kann man sequentiell die Liste mit den einzelnen Bewertungen durchblättern und sich eine Meinung bilden (Abb. 5, B). Diese sequentiellen „Ziehungen" können dann die Grundlage einer erfahrungsbasierten Entscheidung sein. Führen Balkendiagramm und die sequentielle Exposition mit individuellen Bewertungen $\mathrm{zu}$ identischen Entscheidungen? Die verfügbare Evidenz deutet darauf hin, dass Produktentscheidungen auf der Grundlage von Beschreibung bzw. sequentieller Erfahrung die gleichen systematischen Unterschiede aufweisen wie Wahlentscheidungen in Glücksspielen: Seltene Ereignisse (hier sehr positive oder negative Bewertungen) erhalten durch frugale Suche und Recency-Effekt zu wenig Gewicht in erfahrungsbasierten Entscheidungen (vgl. Wulff et al., 2014).

Ist eines der beiden Informationsformate das offensichtlich bessere? Oder anders 
gefragt: Sollte man nicht immer das beschreibungsbasierte Format präferieren, weil es eher vollständige und unverzerrte Information bietet als unsere (begrenzte) Erfahrung? Dass der Sachverhalt nicht so einfach liegt, belegt ein klassischer Befund der Forschung zu Anlegerpräferenzen: Relativ sichere Anlagen - wie zum Beispiel Rentenpapiere - werden trotz schlechterer, langfristiger Renditeerwartungen oft dem Erwerb von Aktien vorgezogen. Der Grund dafür scheint in der psychologischen Überbewertung des kurzfristig hohen Verlustpotentials der Aktien zu liegen (siehe Equity Premium Puzzle; vgl. Bernatzi/Thaler, 1995). Genau an jenem Punkt könnten erfahrungsbasierte Informationsformate von Vorteil sein. Eine aktuelle Studie fand, dass - im Vergleich zur graphischen Beschreibung des Risikos - ein Risiko-Tool, das auf der Präsentation sequentieller Ereignisse beruht und Anlegern die Möglichkeit gibt, Schritt für Schritt Erfahrungen mit der stochastischen Struktur von Aktienpreisen und Renditen zu simulieren, zu mehr Risikobereitschaft und gleichzeitig zu genaueren Einschätzungen des Erwartungswerts und der Wahrscheinlichkeit eines Verlustes führte (vgl. Kaufmann et al., 2013). Eine größere Risikobereitschaft ist natürlich nicht a priori gut und die Anfangsbeispiele legen davon Zeugnis ab. Dennoch ist folgende Überlegung bedeutsam: „The use of experience sampling in financial simulations may be a fruitful strategy for banks to improve the quality of the information they provide about their investment products to ensure that clients understand both the risks they take and the amount of risk they are prepared to take" (Kaufmann et al., 2013, S. 336).

Ähnlich der Entscheidung für die richtige Investitionsanlage hängen viele unternehmerische Entscheidungen von der Kenntnis und Bewertung von Wahrscheinlichkeiten und dem Ausmaß der möglichen Konsequenzen einer Entscheidung ab. Damit drängen sich Implikationen der Kluft zwischen beschreibungsund erfahrungsbasierten Entscheidungen auch für Risikomanagement in Organisationen auf. Viele Alltagsrisiken wie auch Risiken des unternehmerischen Handelns entziehen sich einer einfachen Risikoanalyse. Wahrscheinlichkeiten können oft nicht oder nicht genau quantifiziert werden, weil die in Frage stehenden Ereignisse nicht tabelliert wurden (die Risiken waren nicht als solche erkennbar), singulär sind oder die Stichprobe vergleichbarer Ereignisse sehr klein ist. In Fällen, in denen keine quantifizierten Größen vorliegen, scheint es naheliegend, dass Entscheidungsträger auch ihre persönlichen Erfahrungsstichproben zu Rate ziehen. In diesen Stichproben sind seltene Ereignisse - und dies liegt in der Natur der Sache - in der Regel unterrepräsentiert und werden in erfahrungsbasierten Urteilen vermutlich zu wenig Gewicht erfahren. Allerdings gilt auch dies: Ist ein seltenes Ereignis gerade kürzlich aufgetreten, wird dieses Risiko in erfahrungsbasierten Urteilen für einen gewissen Zeitraum vermutlich zu viel Gewicht erfahren.

Aber selbst wenn das Risikomanagements unternehmerische Chancen und Risiken identifiziert, tabelliert und quantifiziert, stellt sich die Frage, wie sich beschreibungs- und erfahrungsbasierte Beurteilungen zueinander verhalten. Schenken Entscheidungsträger ausschließlich beschreibungsbasierten Analysen von festgestellten Risiken Glauben oder werden diese auch durch die Filter der persönlichen Erfahrungen neubewertet oder gar „verdrängt“? Erste Ergebnisse im Kontext von Warnhinweisen deuten darauf hin, dass bei seltenen Risiken die wiederholte Erfahrung, dass ein Risiko (bislang) nicht eingetreten ist, dazu führen kann, dass es möglicherweise nicht ernst genug genommen wird - ein Verhalten, das insbesondere auch im unternehmerischen Risikomanagement zu folgenschweren Fehleinschätzungen führen kann. Zum Beispiel findet man, dass Patienten ein Medikament mit einer schwerwiegenden, aber seltenen Nebenwirkung auch dann noch einnehmen, wenn vor den Nebenwirkungen gewarnt und das Medikament schlussendlich vom Markt genommen wurde. Die bislang guten Erfahrungen mit dem Medikament vermitteln ein falsches Gefühl der Sicherheit (vgl. Barron et al., 2008). Zweifellos muss die zukünftige Forschung die interessante Interaktion zwischen Erfahrung und Beschreibung in der Bewertung von Risiken weiter ausloten und entschlüsseln.

Schlussendlich sei noch die folgende Überlegung erlaubt: Die Beschreibung der Wahrscheinlichkeit seltener Ereignisse führt eher zu deren Übergewichtung, wohingegen die Erfahrung der Wahrscheinlichkeit seltener Ereignisse eher deren Untergewichtung zur Folge hat. Da- her stellt sich die Frage, ob gute Risikokommunikation - auch im Umfeld unternehmerischer Entscheidungen - darin bestehen könnte und sollte, den Entscheidungsträgern beide Formate zur Verfügung zu stellen. Neben den aggregierten, tabellierten Risiken könnte man "experience sampling“ als Methode verwenden, um - ähnlich wie bei Investigationsentscheidungen (vgl. Kaufmann et al., 2013, S. 336) - Risiken verständlich zu kommunizieren und die Erfahrung eines Risikos unmittelbar zu simulieren.

\section{Fazit}

Bei einer Vielzahl unserer Entscheidungen spielen seltene, aber folgenschwere Ereignisse eine wichtige Rolle. Die bislang etablierte Sichtweise war, dass seltene Ereignisse in Risikowahlen zu viel Gewicht erfahren (gemessen an ihrer objektiven Wahrscheinlichkeit). Diese Sichtweise ist nicht falsch, aber ihr Gültigkeitsbereich ist augenscheinlich begrenzter als bislang vermutet. Der Schlüssel dazu liegt in einer in jüngster Zeit viel beachteten Beobachtung, dem Description-Experience Gap (vgl. Hertwig/Erev, 2009). Der Ausgangspunkt der Forschung zu dieser Kluft ist dieser: Symbolische Informationen über Risiken in Form einer Wahrscheinlichkeit oder einer Aussage begegnen uns allerorts in unserem privaten und professionellen Umfeld. Warnhinweise auf Zigarettenpackungen kommunizieren symbolisch das Risiko des Rauchens, wenngleich in nichtquantifizierter Form: „Raucher sterben früher" oder „Rauchen verursacht tödlichen Lungenkrebs.“ Mediziner kommunizieren die Vorteile und die Risiken von Krebsscreening-Verfahren in Form von deskriptiven statistischen Informationen. Die formalisierte Risikoberichterstattung in Organisationen tut das gleiche, wenn sie den Entscheidungsträgern Risikoberichte als Entscheidungsgrundlage zur Verfügung stellt. Neben der symbolischen Beschreibung von Risiken erfahren Menschen Risiken aber häufig auch durch den Filter ihrer persönlichen Erfahrung. Dem (Risiko-)Controlling kommt in diesem Kontext die Aufgabe zu, die Risikobewertung und damit Entscheidungen zu versachlichen.

Entscheidend ist Folgendes: Viele Untersuchungen zu der Frage, wie Menschen im Angesicht von Risiko und Unsicherheit Entscheidungen treffen, zeigen, dass 
die Vermittlung von relativ unwahrscheinlichen Risiken mittels symbolischer Darstellungen dazu führen kann, dass diesen mehr Gewicht eingeräumt wird als ihnen in Anbetracht ihrer objektiven Wahrscheinlichkeit zusteht. Sobald aber für die Reaktion auf seltene Risiken nicht nur die symbolischen Informationen, sondern auch die eigene Erfahrung herangezogen wird, werden sie relativ angemessen eingeschätzt - zumindest dann, wenn die Erfahrungsstichprobe sehr groß ist. Bei Ereignissen, die so selten sind, dass sie selbst in einer großen Erfahrungsstichprobe nicht auftreten - zum Beispiel eine Weltwirtschaftskrise oder der äußerst seltene Ausbruch eines Vulkans -, neigt unsere begrenzte Erfahrung dazu, das Risiko zu gering zu gewichten. Beschreibung und Erfahrung eines Risikos sind also nicht einfach nur die zwei Seiten derselben Medaille.

Keywords
Decisions from experience and
description
Decisions under risk and uncertainty
Exploration
Psychology of rare events
Risk communication
Summary
This article gives an introduction into
the description-experience gap - the
observation of a systematic difference
in risky choice based on symbolic de-
scriptions versus sequential experi-
ence. The gap is particularly pro-
nounced with rare events. Rare events
appear to receive too much (descrip-
tion) and too little (experience)
weight, respectively. Reasons are dis-
cussed and implications presented.

\section{Literatur}

Barron, G./Leider, S./Stack, J., The effect of safe experience on a warnings' impact: Sex, drugs and rock-n-roll, in: Organizational Behavior and Human Decision Processes, 106. Jg. (2008), H. 2, S. 125-142.

Benartzi, S./Thaler, R. H., Myopic loss aversion and the equity premium puzzle, in: The Quarterly Journal of Economics, 110. Jg. (1995), H. 1, S. 73-92.

Gupta, A. K./Smith, K. G./Shalley, C. E., The Interplay Between Exploration and Exploitation, in: Academy of Management Journal, 49. Jg. (2006), H. 4, S. 693-706.

Hau, R./Pleskac, T. J./Kiefer, J./Hertwig, R., The Description-Experience Gap in Risky Choice: The Role of Sample Size and Experienced Probabilities, in: Journal of Behavioral Decision Making, 21. Jg. (2008), H. 5, S. $493-518$.

Hertwig, R., Decisions from experience. In: Keren, G./Wu, G. (Hrsg.), Blackwell handbook of decision making, im Druck.

Hertwig, R./Barron, G./Weber, E. U./Erev, I., Decisions from experience and the effect of rare events in risky choice, in: Psychological Science, 15. Jg. (2004), H. 8, S. 534-539.

Hertwig R./Erev I., The description-experience gap in risky choice, in: Trends in Cognitive Sciences, 13. Jg. (2009), H. 12, S. 517-523.

Hertwig, R./Pleskac, T. J., Decisions from experience: Why small samples?, in: Cognition, 115. Jg. (2010), H. 2, S. 225-237.

Hills, T. T./Hertwig, R., Information Search in Decisions From Experience Do Our Patterns of Sampling Foreshadow Our Decisions?, in: Psychological Science, 21. Jg. (2010), H. 12, S. 1787-1792.

Kaufmann, C./Weber, M./Haisley, E., The role of experience sampling and graphical displays on one's investment risk appetite, in: Management Science, 59. Jg. (2013), H. 2, S. 323-340.
Lichtenstein, S./Slovic, P./Fischhoff, B./Layman, M./Combs, B., Judged frequency of lethal events, in: Journal of Experimental Psychology: Human Learning \& Memory, 4. Jg. (1978), H. 6, S. 551-578.

de Palma, A./Abdellaoui, M./Attanasi, G./ Ben-Akiva, M./Erev, I./Fehr-Duda, H./Fok, D./Fox, C. R./Hertwig, R./Picard, N./Wakker, P. P./Walker, J. L./Weber, M., Beware of black swans, in: Marketing Letters, im Druck.

Renn, O., Das Risikoparadox: Warum wir uns vor dem Falschen fürchten, Frankfurt a. M. 2014.

Taleb, N. N., The Black Swan: The Impact of the Highly Improbable, New York 2007.

Tversky, A./Kahneman, D., Advances in prospect theory: Cumulative representation of uncertainty, in: Journal of Risk and Uncertainty, 5. Jg. (1992), H. 4, S. 297-323.

Viscusi, W. K., Smoke-filled Rooms: A Postmortem on the Tobacco Deal, University of Chicago Press 2002.

Wulff, D. U./Hills, T. T./Hertwig, R., Online Product Reviews and the Description-Experience Gap, Manuscript submitted for publication 2014.

Literaturtipps aus dem Online-Archiv der Controlling:

- Controlling-Schwerpunkt „Risikomanagement", Ausgabe 1/2013, S. 1-63.

- Julia Gans, Stefan Kreil, Thomas Schild, Effiziente Risikokommunikation für mehr Transparenz und eine risikobewusste Entscheidungsfindung Das Risikomangement der SAP, Ausgabe $4 / 5 / 2012$, S. 237-240.

- Ludwig Sedlmeier, Entscheidungsunterstützung anhand multikriterieller Lösungsverfahren im Controlling, Ausgabe 10/2013, S. 545-547. 